\title{
Calcium Tartrate Tetrahydrate, Case Report of a Novel Human Kidney Stone
}

\author{
Colin Kleinguetl, MD, ScM, James C. Williams, Jr., PhD, Samar A. Ibrahim, MD, \\ Michel Daudon, PhD, Erin T. Bird, MD, and Marawan M. El Tayeb, MD ${ }^{1}$
}

\begin{abstract}
Background: Calcium tartrate tetrahydrate has been reported as the main mineral in urinary stones in rats that have significant tartrate in their diet, but in humans, there has been only one mention of calcium tartrate stones in the form of bladder stone, and that case was in Africa.

Case Presentation: Patient is a 34-year-old Caucasian male who presented with typical symptoms of nephrolithiasis. CT abd/pelvis (renal stone protocol) revealed a $2 \mathrm{~cm}$ nonobstructing stone of the right renal pelvis. Patient underwent an uncomplicated right percutaneous nephrolithotomy and was noted to be stone free after surgery. Stone analysis was difficult with regard to determining composition, but was finally identified as calcium tartrate tetrahydrate.

Conclusion: This was an unusual case, as this is the first recorded case of a calcium tartrate tetrahydrate outside of Africa. This type of stone had only been mainly described in rat models with $d l$-bitartrate in their diet. Our patient was an otherwise healthy, relatively muscular individual with no obvious source for this stone other than a vitamin and amino acid supplement that he takes regularly that contains L-carnitine (as tartrate) and choline (as bitartrate and citrate). The prevalence of this stone type is presently unknown, as stone analysis laboratories have not had the ability to recognize it. Although a connection between the supplement and stone formation is conjecture at this time, we believe this necessitates further investigation.
\end{abstract}

Keywords: urolithiasis, calcium tartrate tetrahydrate, dietary supplement

\section{Introduction and Background}

$\mathbf{U}$ ROLITHIASIS IS A significant clinical issue in both adults and children and its prevalence has been increasing in the United States and across the globe over the last few decades. Overall prevalence varies between studies, but may range from $7 \%$ to $13 \%{ }^{1}$ in North America to $15 \%$ or more. ${ }^{2}$ The most common type of urinary stones are made of calcium, specifically calcium oxalate, ${ }^{3}$ and this is particularly true in the younger age groups. The increased prevalence of stones is certainly multifactorial and may be associated with some lifestyle choices. These choices include poor dietary habits, such as increased consumption of animal protein (and decreased fruits and vegetables), and poor fluid intake that often consists of high sugar beverages. ${ }^{1,2,4}$ Certain supple- ments and vitamins may also play a role in increasing the rate of kidney stones.

In this study, we present a case of urolithiasis that revealed a stone composed of calcium tartrate tetrahydrate, which could have some association with the use of drink supplements containing tartrate. This composition of stone had previously been described mainly in rat models. An association of urolithiasis and renal failure with a purified diet consisting of dl-choline bitartrate was noted in rats by Klurfeld ${ }^{5}$ and Newland et al. ${ }^{6}$ The composition of the stones in these rats was not known until Le Bail et al. ${ }^{7}$ did the crystal determination, confirming the infrared (IR) spectrum published by Newland et al. and identifying the compound as racemic calcium tartrate tetrahydrate, form II.

\footnotetext{
${ }^{1}$ Urology Division, Baylor Scott and White Health, Temple, Texas.

${ }^{2}$ Department of Anatomy and Physiology, Indiana University School of Medicine, Indianapolis, Indiana.

${ }^{3}$ Faculty of Medicine, Alexandria University, Alexandria, Egypt.

${ }^{4}$ Assistance Publique-Hôpitaux de Paris, Hôpital Tenon, Paris, France.
}

(c) Colin Kleinguetl et al. 2017; Published by Mary Ann Liebert, Inc. This is an Open Access article distributed under the terms of the Creative Commons Attribution License, which permits unrestricted use, distribution, and reproduction in any medium, provided the original work is properly cited. 


\section{Presentation of Case}

The patient is a 34-year-old male who initially presented to our clinic for evaluation of recurrent passage of kidney stones. He was initially seen in July 2017 and described the passage of three stones that he was aware of, in the 2 to 3 months leading up to his presentation. During his initial encounter, he described typical symptoms of urolithiasis with primarily right-sided flank pain and intermittent episodes of gross hematuria. Baseline labs were relatively unremarkable (white blood cell [WBC] count 8.2, $\mathrm{Hb} 12.0$ and $\mathrm{Cr} 1.13$ ). Urinalysis demonstrated 2+ blood, 1+ leukocyte esterase, negative nitrites, and 3 to 9 red blood cells and WBCs. Urine culture was negative. Physical examination on presentation was unremarkable.

CT abdomen/pelvis without contrast (renal calculus protocol) was done and demonstrated a $2 \mathrm{~cm}$ nonobstructing stone in the right renal pelvis (Fig. 1). The average Hounsfield unit value was noted to be 825 . The patient was scheduled to undergo right percutaneous nephrolithotomy (PCNL) within 2 weeks of presentation.

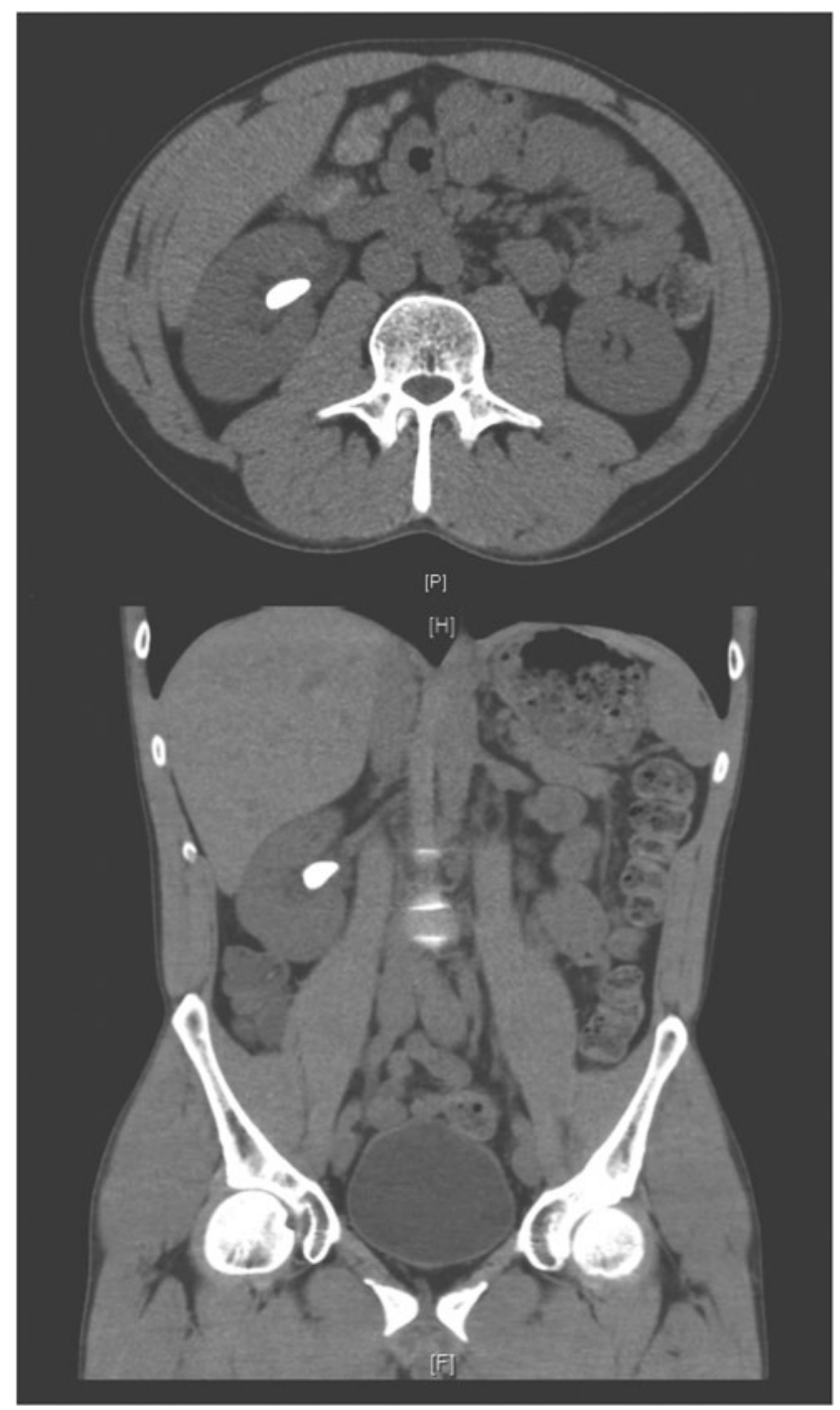

FIG. 1. Preoperative CT abdomen/pelvis (renal stone protocol).

\section{Surgery}

The PCNL procedure was uneventful. A lower pole access was established through biplanar fluoroscopy, access was established on the first try, and the tract was then dilated to 30F using the single step balloon dilation system. Lithotripsy was then achieved using an ultrasonic lithotripter. The stone was noted to be hard, but broke without difficulty. A $10 \mathrm{~F}$ Cope loop nephrostomy tube was left in the upper pole as a nephrostomy tube and a 5F ureteral catheter was left down the ureter to help secure the access. The patient underwent a repeat CT without contrast (renal calculus protocol) the next morning. CT demonstrated the nephrostomy tube in appropriate position and no evidence of residual stone. Hospital course was uncomplicated, right antegrade nephrostogram was done with the contrast seen to be freely flowing down the ureter and the bladder, the nephrostomy tube was removed per protocol, and the patient was discharged on postoperative day 1 .

\section{Postoperative Follow-Up}

UroRisk profile and a future renal ultrasonography (US) were ordered. The renal US was done at 3 weeks was unremarkable,
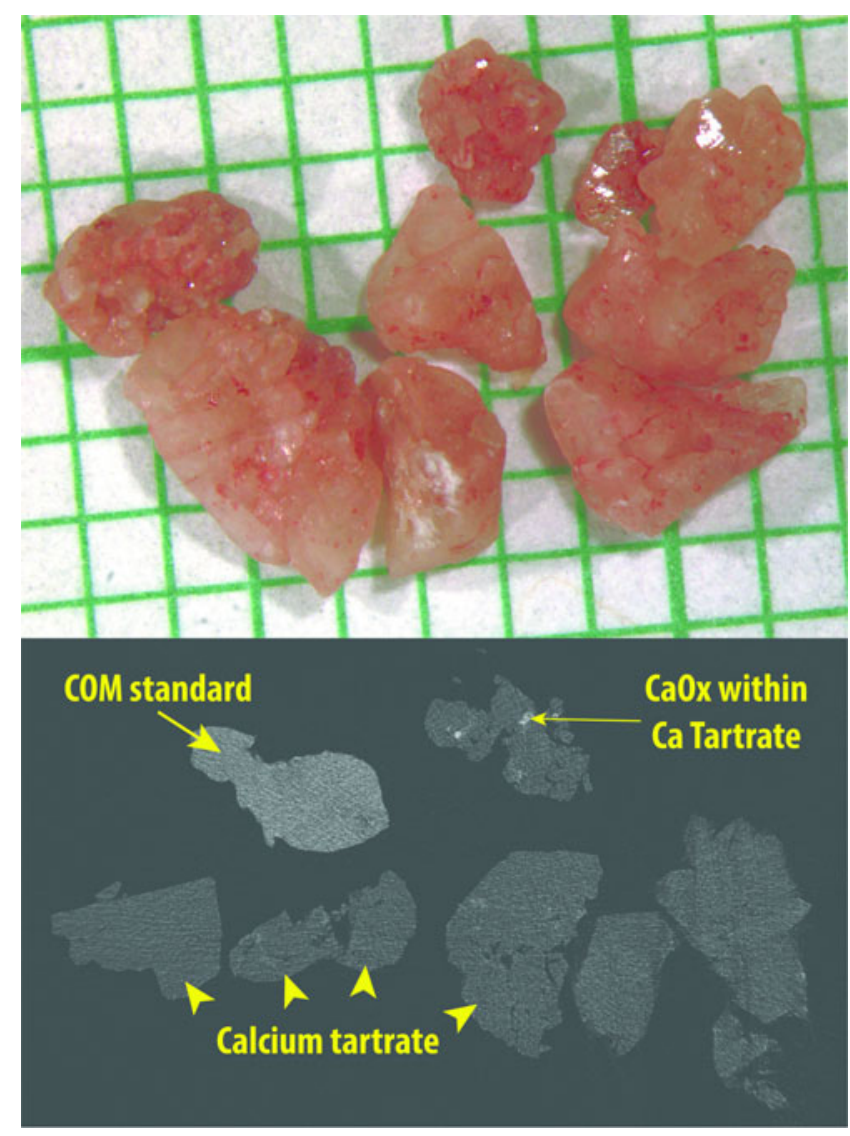

FIG. 2. Photo of stone specimen on mm paper (top), with micro-CT image slice (bottom). Micro-CT slice includes standard specimen composed of pure COM (thick arrow), and apparent $\mathrm{CaOx}$ inclusions in the specimen are indicated with thin arrow. Arrowheads indicate bulk of specimen, which was verified by infrared spectroscopy to be calcium tartrate. $\mathrm{CaOx}$, calcium oxalate; $\mathrm{COM}$, calcium oxalate monohydrate. 
FIG. 3. FT-IR spectrum from the stone specimen. FT-IR, Fourier transform infrared.

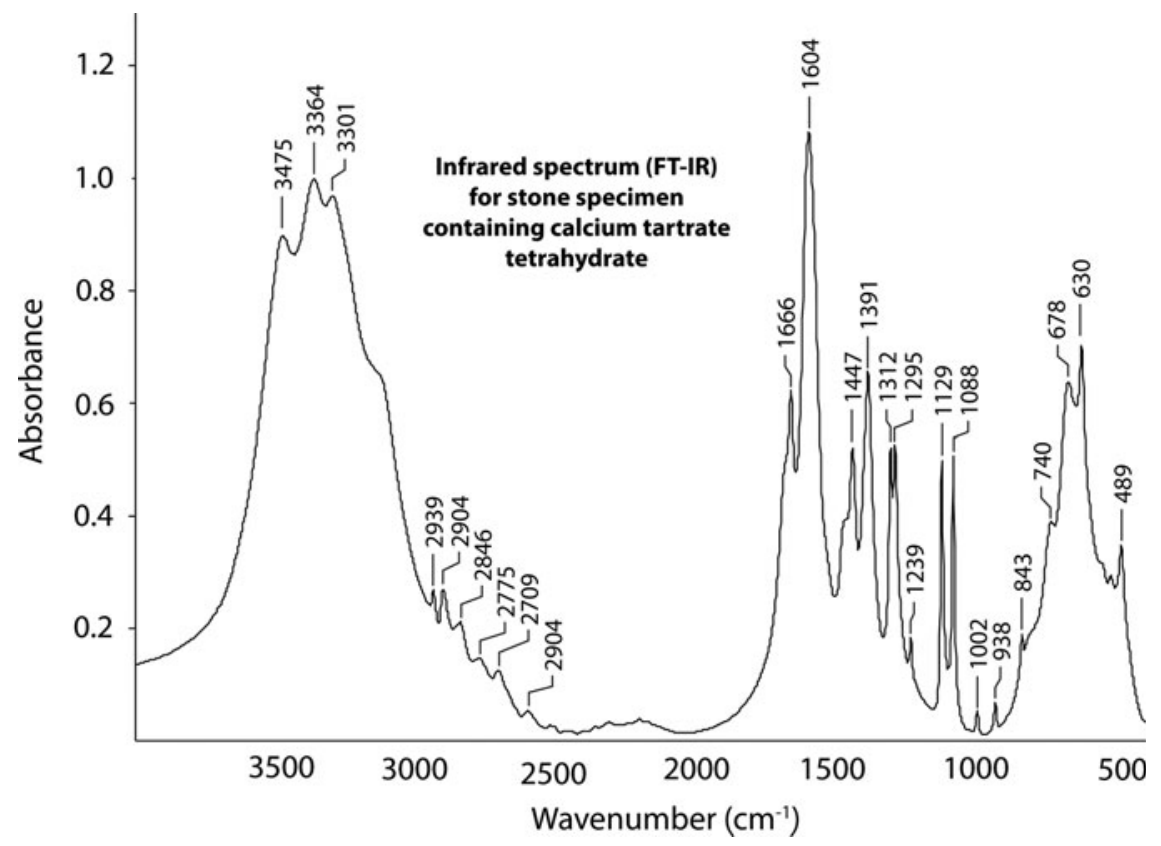

without evidence of obstruction or shadowing to suggest the possible recurrence of stone. Patient was stone free and asymptomatic 10 weeks postoperatively.

\section{Stone Analysis}

The initial stone analysis reported the stone was a $100 \%$ metabolite, composed strictly of Ciprofloxacin, but there was no reason to believe that this was an accurate analysis. The patient was briefly placed on Levofloxacin 2 days before and 5 days after surgery as prophylaxis, but he had no previous history of Ciprofloxacin use. This unusual stone composition resulted in sending the specimen to the stone analysis laboratory at Indiana University, where micro-CT imaging revealed a majority stone material with X-ray attenuation significantly less compared with calcium oxalate (Fig. 2). The IR spectrum of the specimen did not match any known spectrum, and a consult was sought with the laboratory at Tenon Hospital in Paris. That laboratory identified the spectrum as being from calcium tartrate tetrahydrate (Fig. 3).

\section{History/Exposure}

After the finding of calcium tartrate in the stone, a thorough history was obtained. The patient reported no previous history of stones before the events described, no family history of stone formation, and no history of urinary tract infection. With regard to stone formation, he admitted to substantial intake of added salt in his daily diet. The patient worked as a contractor, but only with nonlead-based paints, and there was no apparent exposure to heavy metals or agricultural products. The patient did report drinking "large quantities ...4 or more scoops" of a vitamin and amino acid supplement energy drink/powder every day. Examination of the active ingredients of the product showed the presence of L-carnitine (as tartrate) and choline (as bitartrate and citrate). Each serving (one scoop) contained $10 \mathrm{mg}$ of L-carnitine and
$500 \mathrm{mg}$ choline, and, as such, our patient was drinking 40+ $\mathrm{mg}$ and $2000+\mathrm{mg}$ per day, respectively, with thus an apparent intake of tartrate in the range of grams per day. Currently, there is no FDA-established daily value for intake of any of these substances.

UroRisk profile (24-hour urine study) was largely unrevealing, and the patient had an appropriate total volume of urine, $2.97 \mathrm{~L}$, and a $\mathrm{pH}$ of 6.5. Specifically, there was no evidence of hyperuricemia $(555 \mathrm{mg} /$ day $)$, hypocitraturia (484 mg/day), hyperoxaluria (36 mg/day), or hypercalciuria ( $218 \mathrm{mg} /$ day). Only a mild elevation of urine sodium (216 mEq/day) was present.

\section{Discussion}

Calcium tartrate has been reported previously in only a single person, with data contained only in the tables of an article describing the composition of stones from the West African nation of Burkina Faso. ${ }^{8}$ There are no data in that article on the single male who had a calcium tartrate stone. Before this, calcium tartrate stones had been demonstrated only in rats, in association with tartrate in the diet. ${ }^{5-7}$ The IR spectrum found for the patient in our study (Fig. 3) is strikingly similar to that reported by Newland et al. in their rat study. ${ }^{6}$ The composition of the stone in rats is conclusively calcium tartrate tetrahydrate, ${ }^{7}$ which fits with the presence of tartrate in the diets that caused such stones.

Our patient was a relatively muscular and fit younger male with no significant comorbidities, no significant family history, no known metabolic disorders, and no medications that would be associated with stone formation. As mentioned in his history, he also had no significant chemical exposures, other than the dietary supplement that he took. This supplement contains L-carnitine (as tartrate) and choline (as bitartrate and citrate). Although this is conjecture at this point, as calcium readily binds tartrate, we believe it is reasonable that our patient was ingesting the offending agent, and it was the tartrate in his diet that led to his stones. 


\section{Conclusion}

We report a 34-year-old male with kidney stones composed mainly of calcium tartrate. The patient reported consuming a large amount of tartrate as part of a dietary supplement, which thus could be the cause of his stones. We include the IR spectrum of this patient's stone specimen to facilitate the identification of this unique form of stone in other laboratories.

\section{Acknowledgment}

A special thanks to James Smotherman of Beck Analytical Services for rapid transfer of the stone specimen to Indiana University.

\section{Disclosure Statement}

No competing financial interests exist.

\section{References}

1. Sorokin I, Mamoulakis C, Miyazawa K, et al. Epidemiology of stone disease across the world. World J Urol 2017;35: 1301-1320.

2. Trinchieri A. Epidemiology of urolithiasis: An update. Clin Cases Miner Bone Metab 2008;5:101-106.

3. Daudon M, Donsimoni R, Hennequin C, et al. Sex and agerelated composition of 10617 calculi analyzed by infraredspectroscopy. Urol Res 1995;23:319.

4. Sakhaee K, Maalouf NM, Sinnott B. Clinical review. Kidney stones: Pathogenesis, diagnosis, and management. J Clin Endocrinol Metab 2012;97:1847-1860.

5. Klurfeld DM. Kidney and bladder stones in rodents fed purified diets. J Nutr 2002;132:3785.
6. Newland MC, Reile PA, Sartin EA, et al. Urolithiasis in rats consuming a $d l$ bitartrate form of choline in a purified diet. Comp Med 2005;55:354-367.

7. Le Bail A, Bazin D, Daudon M, et al. Racemic calcium tartrate tetrahydrate [form (II)] in rat urinary stones. Acta Crystallogr B 2009;65:350-354.

8. Dessombz A, Kirakoya B, Coulibaly G, et al. High prevalence of opaline silica in urinary stones from Burkina Faso. Urology 2015;86:1090.

Address correspondence to: Marawan M. El Tayeb, MD Baylor Scott \& White 2401 S. 31st Street Temple, TX 76508

E-mail: Marawan.eltayeb@bswhealth.org

$$
\begin{aligned}
& \text { Abbreviations Used } \\
\mathrm{CT} & =\text { computed tomography } \\
\mathrm{IR} & =\text { infrared } \\
\mathrm{PCNL} & =\text { percutaneous nephrolithotomy } \\
\mathrm{US} & =\text { ultrasonography } \\
\mathrm{WBC} & =\text { white blood cell }
\end{aligned}
$$

Cite this article as: Kleinguetl $\mathrm{C}$, Williams JC, Jr., Ibrahim SA, Daudon M, Bird ET, El Tayeb MM (2017) Calcium tartrate tetrahydrate, case report of a novel human kidney stone, Journal of Endourology Case Reports 3:1, 192-195, DOI: 10.1089/cren.2017.0118. 